\title{
Diagnostic Utility of Salivary Matrix Metalloproteinase-8 (MMP-8) in Chronic Periodontitis: A Novel Approach
}

\author{
A. O. Balogun'1, J. O. Taiwo², O. I. Opeodu², B. F. Adeyemi' , B. M. Kolude ${ }^{3}$ \\ ${ }^{1}$ Department of Family Dentistry, University College Hospital, Ibadan, Nigeria \\ ${ }^{2}$ Department of Periodontology \& Community Dentistry, University of Ibadan/University College Hospital, Ibadan, Nigeria \\ ${ }^{3}$ Department of Oral Pathology, University of Ibadan/University College Hospital, Ibadan, Nigeria \\ Email: josbalo510@gmail.com
}

How to cite this paper: Balogun, A.O., Taiwo, J.O., Opeodu, O.I., Adeyemi, B.F. and Kolude, B.M. (2020) Diagnostic Utility of Salivary Matrix Metalloproteinase-8 (MMP-8) in Chronic Periodontitis: A Novel Approach. Open Journal of Stomatology, 10, $41-49$.

https://doi.org/10.4236/ojst.2020.104006

Received: February 17, 2020

Accepted: March 28, 2020

Published: March 31, 2020

Copyright $\odot 2020$ by author(s) and Scientific Research Publishing Inc. This work is licensed under the Creative Commons Attribution International License (CC BY 4.0).

http://creativecommons.org/licenses/by/4.0/

\begin{abstract}
Currently, diagnosis of chronic periodontitis is based on clinical methods which are cumbersome and have been shown to have inherent errors and drawbacks including inability to detect active disease. There is therefore an urgent need for a timely, cost-effective and less cumbersome method. Salivary matrix metalloproteinase- 8 has been shown in some studies to be a promising bio-marker of chronic periodontitis. This was a case-control study conducted at the University College Hospital, Ibadan. It had 40 cases and 40 controls designed to assess the diagnostic ability of salivary MMP-8 in periodontal disease. Unstimulated whole saliva was assessed using the Quantikine human total MMP-8 ELISA kit from R\&D systems Europe.
\end{abstract}

\section{Keywords}

Saliva, Periodontitis, Diagnosis, MMP-8

\section{Introduction}

Chronic periodontitis is highly prevalent globally especially among the elderly with increased prevalence among Blacks irrespective of their social status [1]. Currently, diagnosis is based on clinical methods, which is cumbersome and has been shown to have inherent errors and drawbacks including inability to detect active disease [2]. Chronic periodontitis was defined by the consensus committee of American Academy of Periodontology (AAP) "as an infectious disease resulting in inflammation within the supporting tissues of the teeth, progressive attachment, and bone loss" [3]. The disease is considered as a polymicrobial in- 
fection involving mainly anaerobic bacteria organized within dental biofilm in the deepened periodontal pocket [4]. At the cellular level, exposure to bacteria and bacterial products such as lipopolysaccharides elicits activation of monocytes/macrophages which encourages secretion of pro-inflammatory mediators such as cytokines, interleukin-1, interleukin-6 (IL6), and Tumor necrotic factor (TNF)- $\alpha$ resulting in the release of matrix metalloproteinases (MMPs) [4] [5]. These are proteinases secreted at the activation of monocyte-macrophage cell line and they belong to a family of structurally related, genetically distinct zinc and calcium dependent enzymes that degrade extracellular matrix and basement membrane. They are also involved in the degradation of several non-extracellular matrixes [4] [5].

Tissue inhibitors of metalloproteinases (TIMPs) regulate the activity of the zinc metalloproteases [5]. TIMPs are naturally occurring endogenous inhibitors to the activities of MMPs, which prevent their hyperactivity. These inhibitors include tissue inhibitors of metalloproteinase 1, 2, 3, and 4 (TIMP-1, TIMP-2, TIMP-3, and TIMP-4). In health, there's a balance between MMPs and TIMPs, which when destabilized, will predispose to destruction of collagen in gingival tissues leading to periodontal tissue destruction [5].

Various authors have established a relationship between Matrix metalloproteinase 8 (MMP-8) and chronic periodontitis [5] [6] [7]. However, due to genetic variations and environmental factors, there is a need to assess the role of this enzyme in the diagnosis of chronic periodontitis among Nigerians. It was therefore the objective of this study to determine the ability of salivary MMP-8 in diagnosing chronic periodontitis in a Nigerian population for the purpose of its utilization as a rapid screening kit in the community.

\section{Objectives}

To assess the diagnostic ability of salivary MMP-8 in periodontal disease.

\section{Methods}

This was a case-control study conducted at the University College Hospital, Ibadan. A representative sample of forty patients with clinical signs of periodontal disease and 40 healthy controls who were age and sex-matched were recruited by simple random sampling. Case and control groups were recruited by balloting from the patients who satisfied the inclusion criteria for both groups within the study period. Participants for the case group were recruited from the Periodontology clinic, University College Hospital, Ibadan while participants for the control were recruited from the Oral Diagnosis Clinic, University College Hospital, Ibadan. The control group included patients who presented for routine dental checkup within the study period. These patients were requested to pick from a ballot box containing wrapped pieces of paper on which "yes" or "no" had been written. For each group, 40 wrapped pieces of paper had "no" and 40 wrapped pieces of paper had "yes". A total of 80 wrapped pieces of paper were in 
each ballot box. Only the respondents who picked "yes" were included in the study.

All participants received full mouth periodontal examination by one calibrated examiner. Before this examination, the medical and dental history were obtained and reviewed along with exclusion criteria. For the purpose of this study, a patient with chronic periodontitis was defined as any individual who had two or more teeth with probing depth (PD) and or clinical attachment loss (CAL) of more than $4 \mathrm{~mm}$ with or without gingival recession. All clinical findings were recorded on the modified WHO oral health assessment form 53 modified to exclude caries, trauma and fluorosis sections.

Participants for both groups were consenting patients $\geq 18$ years who were in good general health with at least 20 erupted teeth. The case group had at least 2 sites with bleeding on probing, probing pocket depth and or clinical attachment loss of greater than $4 \mathrm{~mm}$ with or without gingival recession. Participants in this group were later scheduled for periodontal treatment at no cost.

Participants for the control group had good periodontal health with no bleeding on probing, no periodontal pathologic pocket, no clinical attachment loss and no gingival recession.

Chronic Periodontitis was measured at six locations per tooth namely, mesiobuccal, midbuccal, distobuccal, mesiolingual, distolingual and midlingual surfaces, using the Community Periodontal Index of Treatment Needs (CPITN) periodontal probe and Williams probe for CAL. All the sites were assessed for bleeding on probing, periodontal pathologic pocket and clinical attachment loss.

Unstimulated whole saliva (2 mls) was collected from participants in each group and coded (SP1-40) and (SH1-40) for the case and control groups respectively. The samples were labeled and transported immediately in ice-packs to the laboratory (University College Hospital Virology laboratory) where they were store at $-20^{\circ} \mathrm{C}$ till the time of analysis.

Salivary MMP-8 level was determined in the samples using the Quantikine human total MMP-8 ELISA kit from R\&D systems $s^{\oplus}$ Europe. The Quantikine Human Total MMP-8 Immunoassay utilizes a 4.5 -hours solid phase ELISA designed to measure total MMP-8 (pro- and active MMP-8) in cell culture supernates, serum, plasma, and saliva. It contains NS0-expressed recombinant human MMP-8, and antibodies raised against the recombinant protein. The ELISA kit is all inclusive supplied with optimized reagents ready for use or by simple reconstitution as specified in the manuals provided. R\&D Systems is a well referenced ELISA manufacturer in various studies on salivary MMP-8 [7].

Data was analyzed using SPSS version 22 and presented as frequency tables, graphs, means and standard deviation. T-test was used to compare mean salivary MMP- 8 between the two groups and ROC curve measured diagnostic utility of MMP-8. The level of significance was set at $\mathrm{p}<0.05$. Ethical approval was obtained from the UI/UCH ethical review board with reference number NHREC/5/01/2008a. Informed consent was taken from the patients prior to in- 
clusion in the study.

\section{Result}

A total of eighty respondents, consisting of 35 (43.80\%) females and 45 (56.20\%) males participated in the study. The age range was from 18 - 60 years with a mean of $35.78 \pm 12.46$ years. Majority of the participants, $67 \%$ were married, $25 \%$ were single and $7.5 \%$ were divorced (Table 1 ).

Many of the respondents (52.50\%) had skilled employment, $28.7 \%$ had nonskilled employment while $18.8 \%$ were unemployed.

Frequency distribution of respondents based on their highest academic achievement showed that $37.50 \%$ had tertiary education, $23.80 \%$ had secondary education while $27.5 \%$ had primary education. The others $(11.20 \%)$ had no formal education (Figure 1).

Table 2 illustrates the distribution of clinical attachment loss (CAL) according to their sociodemographic parameters of the study groups. The mean values of clinical parameters of the control and test groups are illustrated in Table 3.

Table 4 demonstrates the mean values of salivary MMP- 8 in the control and test groups. Salivary MMP- 8 concentration was higher in the periodontitis group $(63.74 \pm 27.67 \mathrm{ng} / \mathrm{ml})$ than the control $(16.75 \pm 14.90 \mathrm{ng} / \mathrm{ml})(\mathrm{t}=8.73, \mathrm{p}=$ 0.001). Salivary MMP-8 demonstrated high sensitivity (72\%), specificity (86\%) and diagnostic accuracy with receiver operating characteristic area under curve of 0.78 (Figure 2). Analysis of the ROC curve is presented in Table 5.

Table 1. Sociodemographics.

\begin{tabular}{ccc}
\hline Socio-demographic characteristics & Frequency & Percentage \\
\hline Age [Years] & 35 & 43.7 \\
$18-30$ & 14 & 17.5 \\
$31-40$ & 31 & 38.8 \\
$>40$ & & \\
Gender & 45 & 56.3 \\
Male & 35 & 43.7 \\
Female & & \\
Marital status & 20 & 25 \\
Single & 54 & 67.5 \\
Married & 6 & 7.5 \\
Divorced & & 52.5 \\
Occupation & 15 & 28.7 \\
Skilled & 42 & 18.8 \\
Unskilled & 23 & \\
Unemployed & & \\
\hline & & \\
\hline & & \\
\hline
\end{tabular}


Table 2. Impact of age, gender and level of education on chronic periodontitis.

\begin{tabular}{ccccc}
\hline \multicolumn{1}{c}{ Variables } & & Periodontal Index [CAL] & Test & P-Value \\
\hline \multirow{2}{*}{ Gender } & Male & $2.82[2.62 \mathrm{SD}]$ & $\mathrm{t}=0.163$ & 0.688 \\
& Female & $2.57[2.69 \mathrm{SD}]$ & & \\
& $18-30$ & $2.31[2.70 \mathrm{SD}]$ & $\mathrm{F}=-2.465$ & 0.016 \\
Age Groups [years]: & $31-43$ & $2.71[2.73 \mathrm{SD}]$ & & \\
& 43 and above & $3.16[2.83 \mathrm{SD}]$ & & 0.017 \\
& No & $4.67[2.29 \mathrm{SD}]$ & $\mathrm{F}=-2.430$ & \\
Level of Education & Primary & $2.79[2.76 \mathrm{SD}]$ & & \\
& Secondary & $2.27[2.83 \mathrm{SD}]$ & & \\
& Tertiary & $2.40[2.66 \mathrm{SD}]$ & & \\
\hline
\end{tabular}

Table 3. Frequency distribution of mean values of clinical parameters of respondents.

\begin{tabular}{ccc}
\hline $\begin{array}{c}\text { Clinical parameters of periodontal } \\
\text { disease }\end{array}$ & Experimental group[Mean] & Control [Mean] \\
\hline Gingival index at baseline & $2.10 \pm 0.47$ & 0.00 \\
Oral hygiene index & $4.20 \pm 0.50$ & 0.05 \\
Probing depth at baseline & $5.87 \pm 0.91$ & $2.45 \pm 0.5$ \\
Clinical attachment loss at baseline & $5.40 \pm 0.74$ & 0.00 \\
CPITN Code at baseline & $3.10 \pm 0.49$ & 0.00 \\
\hline
\end{tabular}

Table 4. Mean salivary MMP-8 of case and control groups.

\begin{tabular}{cccccc}
\hline & $\begin{array}{c}\text { Case group } \\
{[\mathrm{ng} / \mathrm{ml}]}\end{array}$ & $\begin{array}{c}\text { Control group } \\
{[\mathrm{ng} / \mathrm{ml}]}\end{array}$ & $\begin{array}{c}\text { Student } \\
\text { t-test }\end{array}$ & $\begin{array}{c}\text { Deg. of } \\
\text { freedom }\end{array}$ & p-value \\
\hline MMP-8 & $63.74 \pm 27.68$ & $16.75 \pm 14.9$ & 8.73 & 39 & 0.001 \\
\hline
\end{tabular}

Table 5. Receiver operating characteristics [ROC] curve.

\begin{tabular}{ccccc}
\hline \multirow{2}{*}{$\begin{array}{c}\text { Area under the } \\
\text { curve }\end{array}$} & Standard Error & P-value & \multicolumn{2}{c}{ 95\% Confidence Interval } \\
\cline { 3 - 4 } & 0.054 & 0.001 & Lower Bound & Upper Bound \\
\hline 0.783 & 0.0578 & 0.888 \\
\hline
\end{tabular}

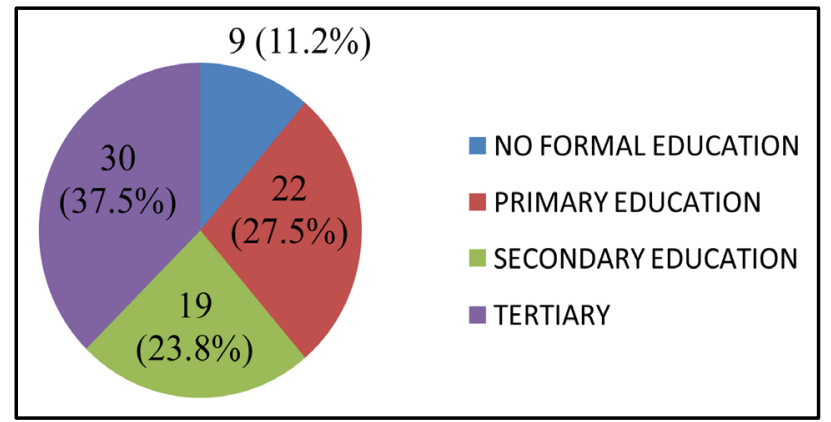

Figure 1. Frequency distribution of respondents according to their highest academic status. 


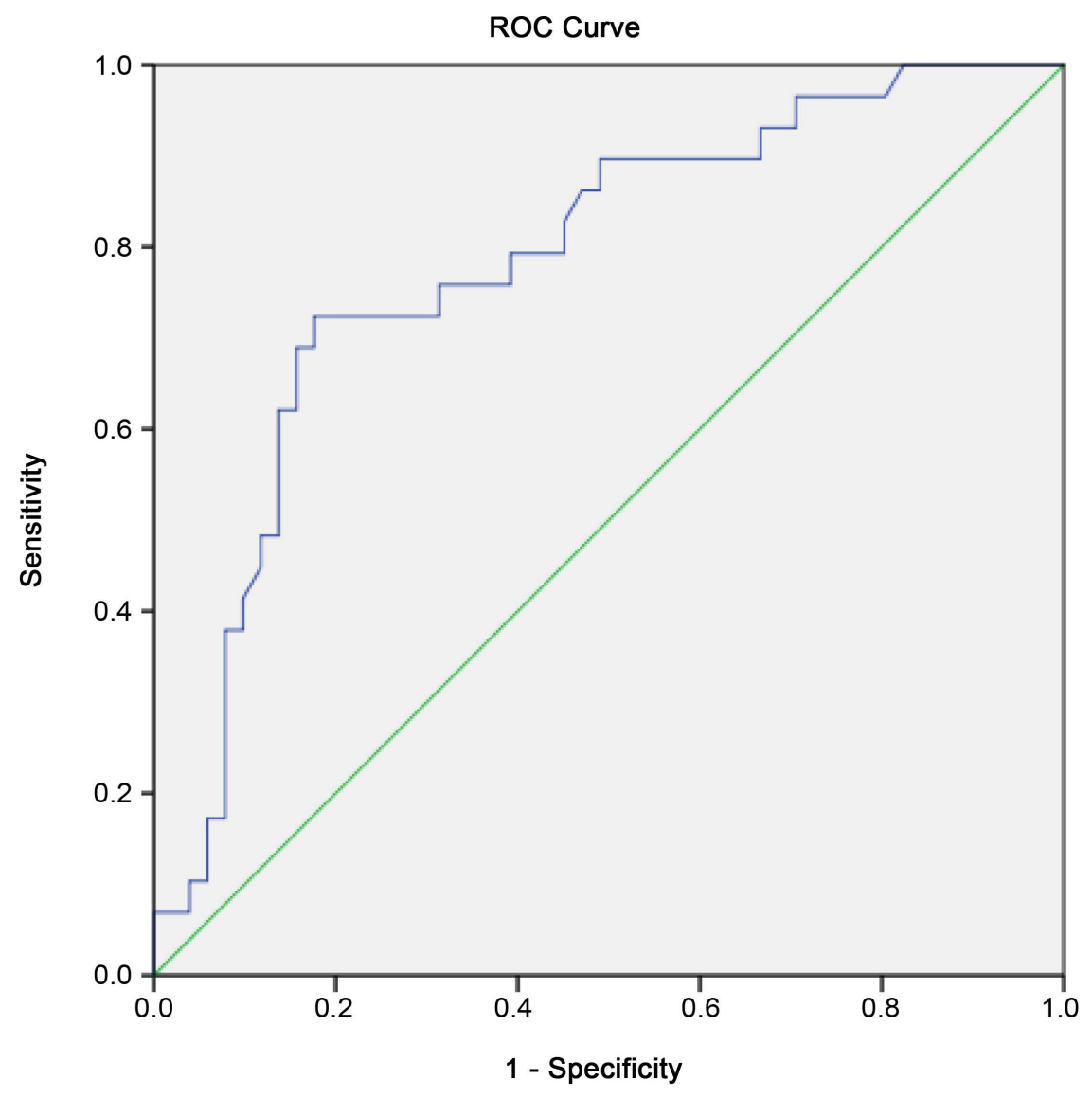

Diagonal segments are produced by ties.

Figure 2. Receiver operating characteristic (ROC) analysis of salivary mmp-8 and periodontitis.

\section{Discussion}

Periodontal disease is an inflammatory disease affecting the soft and hard tissues that support the teeth [4]. It is a disease of great public health concern due to its effect on patient's quality of life [8]. Oral health (including periodontal health) has been described as an integral aspect of general health and a bi-directional relationship has been established between periodontal disease and some non-communicable diseases NCDs [8]. Therefore, its early detection and treatment have become ever important.

Active surveillance of periodontal disease is practically non-existent in Nigeria due to the cumbersome nature of the existing clinical methods which are tedious and time-consuming [4]. These methods also lack the ability to detect active disease. Chronic periodontitis has been associated with the non-communicable diseases such as diabetes mellitus, cardiovascular disease and so on (NCDs) [9]. Therefore, its early detection and treatment is very important. This study has brought to fore, the ability of salivary MMP- 8 to distinguish patients with periodontal disease from periodontally healthy patients. The mean salivary MMP-8 value of $63.74 \pm 27.68 \mathrm{ng} / \mathrm{dl}$ was seen in the case group and a mean salivary 
MMP-8 of $16.75 \pm 14.90 \mathrm{ng} / \mathrm{dl}$ was seen in the control group (Table 4). The salivary MMP-8 levels were seen to be about four times higher in the periodontally diseased group and showed statistical significance $(\mathrm{p}=0.001)$ between the two groups (Table 4). This relationship between salivary MMP-8 and periodontal disease was established many years ago and confirmed by various studies including this study [7] [10] [11].

This study also corroborates an earlier study done by Fenol et al. (2014) where the salivary levels of MMP-8 were found to be $94.3 \pm 32.5 \mathrm{ng} / \mathrm{ml}$ in the periodontally diseased group and $77.6 \pm 25.3 \mathrm{ng} / \mathrm{ml}$ in the healthy control group [5]. Other similar studies have also substantiated these findings. These include Gupta 2015 [6], Dayanand et al. 2014 [10] and Mwai 2014 [7]. Genetic and racial differences in the Nigerian population may be responsible for the varied mean concentrations of salivary MMP- 8 between the aforementioned studies. Studies have indicated the potential utility of saliva as a diagnostic fluid as well as in monitoring therapeutic interventions [12] [13] [14].

Receiver operating characteristic (ROC) curve values may vary between studies [15]. The curve is an established technique in comparing diagnostic tests [15]. Figure 2 illustrates the ROC curve which demonstrated that salivary MMP-8 could distinguish between individuals with and without periodontitis with an area under the curve [AUC] value of 0.78 . This was in agreement with a recent study on salivary biomarkers by Ebersole and colleagues who established an AUC value of 0.7 using salivary MMP-8 [12]. Similar findings were also observed in a study by Ramseier, and colleagues where an AUC of 0.9 was derived [13]. The more robust AUC value in the Ramseier study was possibly due to the analysis of a combination of biomarkers (MMP-8, MMP-9 and osteoprotegerin) as well as genomic identification of red complex periodontal pathogens.

The high prevalence of periodontitis and its impact on cardiovascular diseases and NCDs makes its surveillance of utmost importance [16]. However, present clinical methods are cumbersome [2]. The high diagnostic accuracy of MMP-8 will encourage its use as a screening tool especially in the community and by non-dental personnel.

In this study, diverse and varied ages of adults sought dental treatment at the study area. This is reflected in the mean age (35.78 years and a broad standard deviation of 12.46). This study confirms the higher prevalence of periodontal disease in males than females because many of the participants were males. Also, severity of periodontal disease as measured by the CAL was worse in the male gender than the females as shown by the higher mean CAL $(2.82 \pm 2.62)$. This was however not statistically significant $(\mathrm{p}=0.69)$. A previous study has associated the better periodontal status in the females to the better oral hygiene in them. [1]. The mean clinical attachment loss also tends to be worse with the increasing age of respondents, which some studies have reportedly claimed to be due to increased susceptibility of periodontal tissue with increasing age (Table 3) [17]. However, it has been reported that the increased attachment loss seen 
with the increasing age could be due to the individual host susceptibility to periodontal disease rather than the length of time the plaque is present (age effect) [4] [17]. The greater the susceptibility of an individual to the disease, the slower the rate of wound healing and the faster the periodontal disease tend to progress [4] [17].

It is important to stress that the use of the clinical methods of diagnosis (measuring CAL, PD and bleeding on probing) may still be important in individuals with high levels of salivary MMP-8 to further tests for chronic periodontitis. The cost of the screening kit and its non-availability may be a limitation to the use of this biomarker in periodontal diagnosis.

\section{Conclusion}

Salivary MMP-8 demonstrated high diagnostic accuracy in the study group. This suggests that MMP- 8 can play useful role in the diagnosis of periodontitis.

\section{Recommendation}

Clinicians and community health workers should be encouraged to employ the use of MMP-8 kit for rapid screening of periodontal disease.

\section{Conflicts of Interest}

The authors declare no conflicts of interest regarding the publication of this paper.

\section{References}

[1] Taiwo, J.O., Jeboda, S.O., Motayo, T.O. and Obiechina, A.E. (2004) Periodontal Health of the Elderly People in South East Local Government Area in Ibadan, Nigeria. The African Journal of Medical Sciences, 33, 285-291.

[2] Savage, A., Eaton, K.A., Moles, D.R. and Needleman, I.A. (2009) Systemic Review of Definitions of Periodontitis and Methods That Have Been Used to Identify This Disease. Journal of Clinical Periodontology, 36, 458-467. https://doi.org/10.1111/j.1600-051X.2009.01408.x

[3] Armitage, G.C. (1999) Development of a Classification System for Periodontal Disease and Conditions. Annals of Periodontology, 4, 1-6.

[4] Newman, G.M., Takei, H.H. and Carranza, F. (2012) Carranza's Clinical Periodontology. Elsevier Inc., Amsterdam, 203-205.

[5] Fenol, A., Peter, M.R., Perayil, J., Vyloppillil, R. and Bhaskar, A. (2014) Comparison of Salivary TIMP-1 Levels in Periodontally Involved and Healthy Controls and the Response to Nonsurgical Periodontal Therapy. International Journal of Chronic Diseases, 2014, Article ID: 363581. https://doi.org/10.1155/2014/363581

[6] Gupta, N., Gupta, N.D., Gupta, A., Khan, S., et al. (2015) Role of Salivary Matrix Metalloproteinase-8 (MMP-8) in Chronic Periodontitis Diagnosis. Frontiers in Medicine, 9, 72-76. https://doi.org/10.1007/s11684-014-0347-x

[7] George Karimi Mwai (BDS) (2015) Salivary Matrix Metalloproteinase-8 Levels and Periodontal Health Status among Adults Attending the University of Nairobi Dental Hospital. 
[8] Llewellyn, C.D. and Warnakulasuriya, S. (2003) The Impact of Stomatological Disease on Oral Health-Related Quality of Life. European Journal of Oral Sciences, 111, 297-304. https://doi.org/10.1034/j.1600-0722.2003.00057.x

[9] Stathopouloue, P.G., Kinane, D.F. and Peterson, M. (2000) Environmental and Other Modifying Factors of the Periodontal Diseases. Periodontology, 40, 107-119. https://doi.org/10.1111/j.1600-0757.2005.00136.x

[10] Dayanand, J.V., Sangeetha, S.P. and Ponnudurai, S.G. (2014) Assessment of Matrix Metalloproteinases-8 and -9 in Gingival Crevicular Fluid of Smokers and Non-Smokers with Chronic Periodontitis Using ELISA. Journal of International Oral Health, 6, 67-71.

[11] Sorsa, T., Tervahartiala, T., Leppilahti, J., Hernandez, M., Gamonal, J., et al. (2011) Collagenase-2 (MMP-8) as a Point-of-Care Biomarker in Periodontitis and Cardiovascular Diseases. Pharmacological Research, 63, 108-113.

https://doi.org/10.1016/j.phrs.2010.10.005

[12] Ebersole, J.L., Schuster, J.L., Stevens, J., Dawson, D., Kryscio, R.J., et al. (2013) Patterns of Salivary Analytes Provide Diagnostic Capacity for Distinguishing Chronic Adult Periodontitis from Health. Journal of Clinical Immunology, 33, 271-279. https://doi.org/10.1007/s10875-012-9771-3

[13] Ramseier, C.A., Kinney, J.S., Herr, A.E., Braun, T., Sugai, J.V., Shelburne, C.A., et al. (2009) Identification of Pathogen and Host-Response Markers Correlated with Periodontal Disease. Journal of Periodontology, 80, 436-446. https://doi.org/10.1902/jop.2009.080480

[14] Rathnayake, N., Åkerman, S., Klinge, B., Lundegren, N., Jansson, H., Tryselius, Y., et al. (2013) Salivary Biomarkers of Oral Health-A Cross-Sectional Study. Journal of Clinical Periodontology, 40, 140-147. https://doi.org/10.1111/jcpe.12038

[15] Zweig, M.H. and Campbell, G. (1993) Receiver-Operating Characteristic (ROC) Plots: A Fundamental Evaluation Tool in Clinical Medicine. Clinical Chemistry, 39, 561-577. https://doi.org/10.1093/clinchem/39.4.561

[16] Eke, P.I., Dye, B.A., Wei, L., et al. (2012) CDC Periodontal Disease Surveillance Workgroup. Journal of Dental Research, 91, 914-920. https://doi.org/10.1177/0022034512457373

[17] Van der Velden, U. (1984) Effect of Age on the Periodontium. Journal of Clinical Periodontology, 11, 281-294. https://doi.org/10.1111/j.1600-051X.1984.tb01325.x 